\title{
THREE-NUCLEON SCATTERING AT INTERMEDIATE ENERGIES
}

\author{
I. FACHRUDDIN \\ Departemen Fisika, Universitas Indonesia, Depok 16424, Indonesia \\ CH. ELSTER \\ Department of Physics and Astronomy, Ohio University, Athens, OH 34701, USA \\ W. GLÖCKLE \\ Institut für Theoretische Physik II, Ruhr-Universität Bochum, D-44780 Bochum, \\ Germany
}

\begin{abstract}
By means of a technique, which does not employ partial wave (PW) decompositions, the nucleon-deuteron break-up process is evaluated in the Faddeev scheme, where only the leading order term of the amplitude is considered. This technique is then applied to calculate the semi-exclusive proton-deuteron break-up reaction $d(p, n) p p$ for proton laboratory energies $E_{l a b}$ of a few hundred $\mathrm{MeV}$. A comparison with PW calculations is performed at $197 \mathrm{MeV}$ projectile energy. At the same energy rescattering processes, which are not included in the 3D calculations yet, are shown to be still important in the full Faddeev PW calculations, especially for the cross section and the analyzing power $A_{y}$. Next kinematical relativistic effects are investigated for projectile energies up to about $500 \mathrm{MeV}$. At the higher energies, those relativistic effects start not to be negligible, especially in the peak of the cross section.
\end{abstract}

\section{Introduction}

In order to investigate the short distance behavior of three nucleon $(3 \mathrm{~N})$ as well as nucleon-nucleon (NN) forces it is necessary to consider $3 \mathrm{~N}$ scattering at higher energies of a few hundred $\mathrm{MeV}$. The partial wave (PW) technique, which takes as basis states a specific number of angular momentum eigenstates, has a long history of being employed to solve Faddeev equations for $3 \mathrm{~N}$ scattering [1]. However as the energy increases the number of contributing angular momenta proliferates, leading to increased algorithmic difficulties as well as more tedious algebraic work. Therefore, a new and alternative approach is needed, which is not based on PW basis.

In momentum space the choice is to work directly with momentum vector states. For three boson scattering this approach has been pioneered successfully carried out in Refs. 2]. We develop our approach [3], referred here as the 3D technique, for three nucleon scattering, allowing the use of realistic nuclear forces, since spin and isospin are also taken into account. Since the NN system serves as input for our 
$3 \mathrm{~N}$ calculations, we successfully applied the $3 \mathrm{D}$ technique to both $\mathrm{NN}$ scattering [4] and the deuteron $[5$.

We evaluate the nucleon-deuteron (Nd) break-up process using the Faddeev scheme. However, we do not solve the full Faddeev equations but rather concentrate on the first order term in the multiple scattering series given by them. Thus, we assume that at intermediate energies of a few hundred $\mathrm{MeV}$ the leading term may sufficiently describe the scattering process. We include relativistic effects in the kinematics to investigate their size as function of energy. For our application we concentrate on the semi-exclusive $d(p, n) p p$ reaction and calculate the spin averaged differential cross section, the neutron polarization, the proton analyzing power and the polarization transfer coefficients. There are in fact experimental data in the energy range up to about $500 \mathrm{MeV}[6-[8]$. The 3D technique works with any potentials given in operator form, not the PW projected ones. We employ the NN potentials AV18 [9] and Bonn-B [10].

\section{The Nd Break-Up Amplitude}

The break-up process is given by the Nd break-up operator $U_{0}$ given by

$$
U_{0}=(1+P) T P
$$

where $P \equiv P_{12} P_{23}+P_{13} P_{23}$ is a permutation operator, $T$ stands for NN t-matrix. The term $T P$ is the leading term of the $3 \mathrm{~N}$ transition operator $T_{F}$ obeying Faddeev equation $T_{F}=T P+T G_{0} P T_{F}$, with $G_{0}$ being the free three-nucleon propagator. The operator $U_{0}$ is fully anti-symmetrized and can be written as a sum of three terms in $T P$, the first of which is $U_{0}^{(1)}=T P$. The other terms $U_{0}^{(2)}=P_{12} P_{23} T P$ and $U_{0}^{(3)}=P_{12} P_{23} T P$ are related to $U_{0}^{(1)}$ by means of permutations.

Without employing PW decompositions, except for the deuteron, the Nd breakup amplitude $U_{0}(\mathbf{p}, \mathbf{q})$ is given in Eq. (2) (see Ref. 3] for derivation), where $m_{s i}, \tau_{i}$ $(i=1,2,3)$ are the spins and isospins of the three nucleons in final state, $m_{s 1}^{0}, \tau_{1}^{0}$ the spin and isospin of nucleon 1 acting as the projectile, $\Psi_{d}^{M_{d}}$ the deuteron state, $M_{d}$ the projection of total angular momentum of the deuteron along an arbitrary z-axis, $\psi_{l}\left(\pi^{\prime}\right)$ the deuteron wave function components (s and d waves), $T_{\Lambda \Lambda^{\prime}}^{\pi S t}\left(p, \pi, \cos \theta^{\prime} ; E_{p}\right)$ the NN t-matrix elements in a momentum-helicity basis [4] for given parity $\pi$, total spin $S$ and isospin $t$, final and initial helicities $\Lambda$ and $\Lambda^{\prime}$, and a center of mass energy $E_{p}$ of the 23-subsystem, $d_{\Lambda^{\prime} \Lambda}^{S}(\theta)$ 's being the rotation matrices [11. The Jacobi momenta $\mathbf{p}$ and $\mathbf{q}$ describe the $3 \mathrm{~N}$ kinematics in the final state, where $\mathbf{p}$ is the relative momentum between nucleon 2 and $3, \mathbf{q}$ the relative momentum of nucleon 1 to the 23 -subsystem, and $\mathbf{q}_{0}$ the relative momentum of the projectile to the deuteron.

The operator $U_{0}(\mathbf{p}, \mathbf{q})$ given in Eq. (2) is derived within the framework of the nonrelativistic Faddeev scheme. By adopting the formulation given in Ref. [12, relativistic kinematics is introduced. Thus, we re-evaluate the Jacobi momenta, carry out corresponding Lorentz transformations to the two- and three-particle c.m. 
subsystems, and employ relativistic energy-momentum relations. To calculate the observables, a relativistic description of the cross section is employed.

$$
\begin{aligned}
& U_{0}(\mathbf{p}, \mathbf{q}) \equiv\left\langle\mathbf{p q} m_{s 1} m_{s 2} m_{s 3} \tau_{1} \tau_{2} \tau_{3}\left|U_{0}\right| \mathbf{q}_{0} m_{s 1}^{0} \tau_{1}^{0} \Psi_{d}^{M_{d}}\right\rangle \\
& =\frac{(-)^{\frac{1}{2}+\tau_{1}}}{4 \sqrt{2}} \delta_{\tau_{2}+\tau_{3}, \tau_{1}^{0}-\tau_{1}} \sum_{m_{s}^{\prime}} e^{-i\left(\Lambda_{0} \phi_{p}-\Lambda_{0}^{\prime} \phi_{\pi}\right)} C\left(\frac{1}{2} \frac{1}{2} 1 ; m_{s}^{\prime} m_{s 1}\right) \\
& \times \sum_{l} C\left(l 11 ; M_{d}-m_{s}^{\prime}-m_{s 1}, m_{s}^{\prime}+m_{s 1}\right) Y_{l, M_{d}-m_{s}^{\prime}-m_{s 1}}\left(\hat{\pi}^{\prime}\right) \psi_{l}\left(\pi^{\prime}\right) \\
& \times \sum_{S \pi t}\left(1-\eta_{\pi}(-)^{S+t}\right) C\left(\frac{1}{2} \frac{1}{2} t ; \tau_{2} \tau_{3}\right) C\left(\frac{1}{2} \frac{1}{2} t ; \tau_{1}^{0},-\tau_{1}\right) \\
& \times C\left(\frac{1}{2} \frac{1}{2} S ; m_{s 2} m_{s 3} \Lambda_{0}\right) C\left(\frac{1}{2} \frac{1}{2} S ; m_{s 1}^{0} m_{s}^{\prime} \Lambda_{0}^{\prime}\right) \\
& \times \sum_{\Lambda \Lambda^{\prime}} d_{\Lambda_{0} \Lambda}^{S}\left(\theta_{p}\right) d_{\Lambda_{0}^{\prime} \Lambda^{\prime}}^{S}\left(\theta_{\pi}\right) e^{i\left(\Lambda^{\prime} \phi^{\prime}-\Lambda \Omega\right)} T_{\Lambda \Lambda^{\prime}}^{\pi S t}\left(p, \pi, \cos \theta^{\prime} ; E_{p}\right) \\
& +U_{0}^{(2)}(\mathbf{p}, \mathbf{q})+U_{0}^{(3)}(\mathbf{p}, \mathbf{q}),
\end{aligned}
$$

with

$$
\begin{aligned}
\boldsymbol{\pi} & \equiv \frac{1}{2} \mathbf{q}+\mathbf{q}_{0} \quad \boldsymbol{\pi}^{\prime} \equiv-\mathbf{q}-\frac{1}{2} \mathbf{q}_{0} \\
\cos \theta^{\prime} & =\cos \theta_{p} \cos \theta_{\pi}+\sin \theta_{p} \sin \theta_{\pi} \cos \left(\phi_{p}-\phi_{\pi}\right) \\
e^{i\left(\Lambda^{\prime} \phi^{\prime}-\Lambda \Omega\right)} & =\frac{\sum_{N=-S}^{S} e^{i N\left(\phi_{p}-\phi_{\pi}\right)} d_{N \Lambda}^{S}\left(\theta_{p}\right) d_{N \Lambda^{\prime}}^{S}\left(\theta_{\pi}\right)}{d_{\Lambda^{\prime} \Lambda}^{S}\left(\theta^{\prime}\right)} .
\end{aligned}
$$

\section{The Semi-exclusive Proton-Deuteron Break-Up Reaction}

Our formulation is applied to the semi-exclusive proton-deuteron (pd) break-up reaction $d(p, n) p p$. We calculate the spin averaged differential cross section, the neutron polarization, the proton analyzing power and the polarization transfer coefficients for proton laboratory energies $E_{l a b}$ up to about $500 \mathrm{MeV}$.

First, we perform comparisons with calculations based on the well established PW technique, which also include only the leading term of the Faddeev amplitude. Both schemes agree for projectile energies $E_{l a b}<200 \mathrm{MeV}$. However, at about 200 $\mathrm{MeV}$ deviations occur in the cross section peak, as shown in Fig. 1 for the cross section at $E_{l a b}=197 \mathrm{MeV}$, where the PW calculation has not sufficiently converged to the 3D calculation. The PW calculation shown in Fig. 1 takes into account NN angular momenta up to $j=5$ and 7 , and $3 \mathrm{~N}$ states for total $3 \mathrm{~N}$ angular momenta up to $J=31 / 2$, which is a technical maximum at present.

Since we do not take rescattering terms into account, we nevertheless have to estimate their effects. At $E_{l a b}=197 \mathrm{MeV}$ we do this by comparing the 3D calculation with a full Faddeev PW calculation, and display the results in Fig. 2. We see that the higher order multiple scattering contributions lower the cross section and 


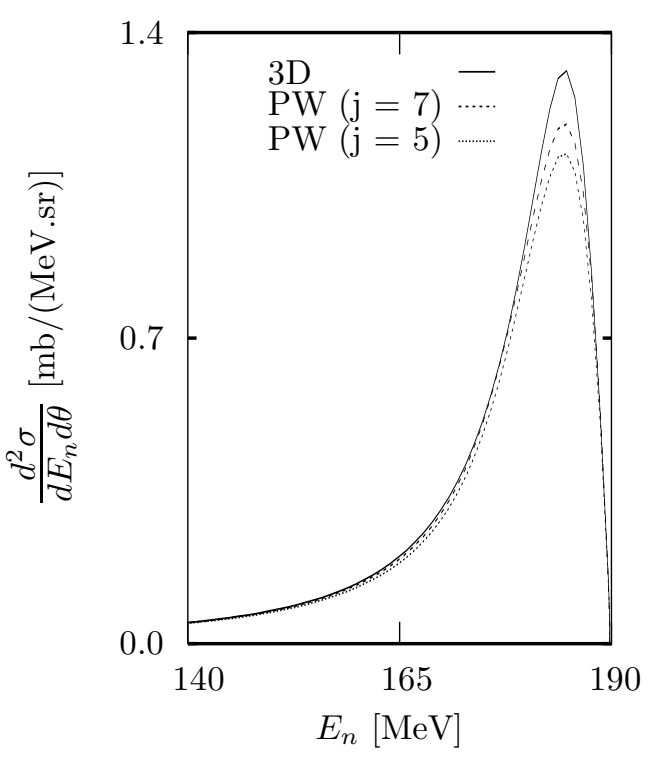

Figure 1. The spin averaged differential cross section at $E_{l a b}=197 \mathrm{MeV}, \theta=13^{0}$, based on the Bonn-B potential.

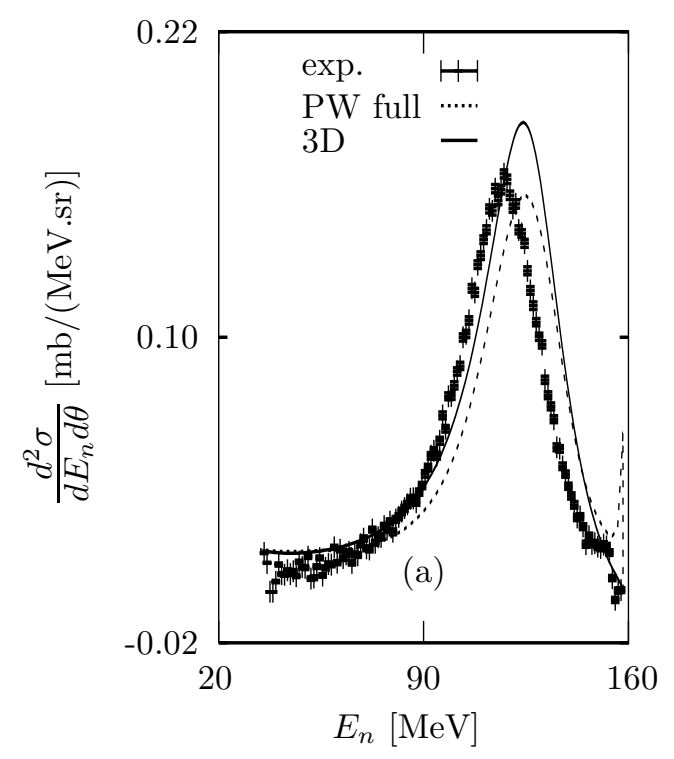

are essential for the description of the analyzing power especially for the small energies of the outgoing neutron.

Next, we investigate effects of relativistic kinematics on semi-inclusive observables as function of the projectile energy. In Fig. 3 we compare our nonrelativistic $3 \mathrm{D}$ calculations with the corresponding relativistic ones, and see that the position as well as the height of the quasi-free peak are influenced. Since the position of the quasi-free peak is solely determined by kinematics, it is satisfying to see that the use of relativistic kinematics put the calculated peak at the right position with respect to the data. As expected the effect of relativistic kinematics increases with increasing projectile energy.

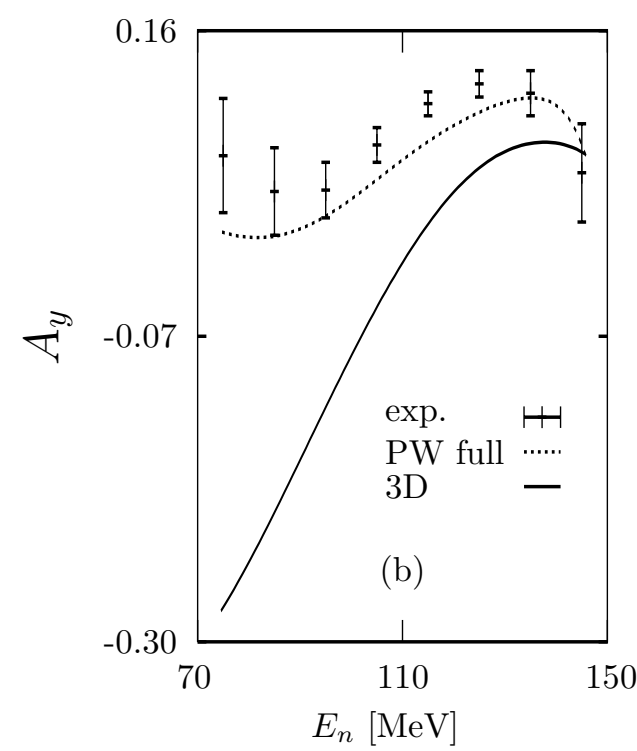

Figure 2. The spin averaged differential cross section (a) and the analyzing power $A_{y}$ (b) at $E_{l a b}=197 \mathrm{MeV}, \theta=37^{\circ}$. The calculations are based on the AV18 potential, the data from Ref. [8]. 

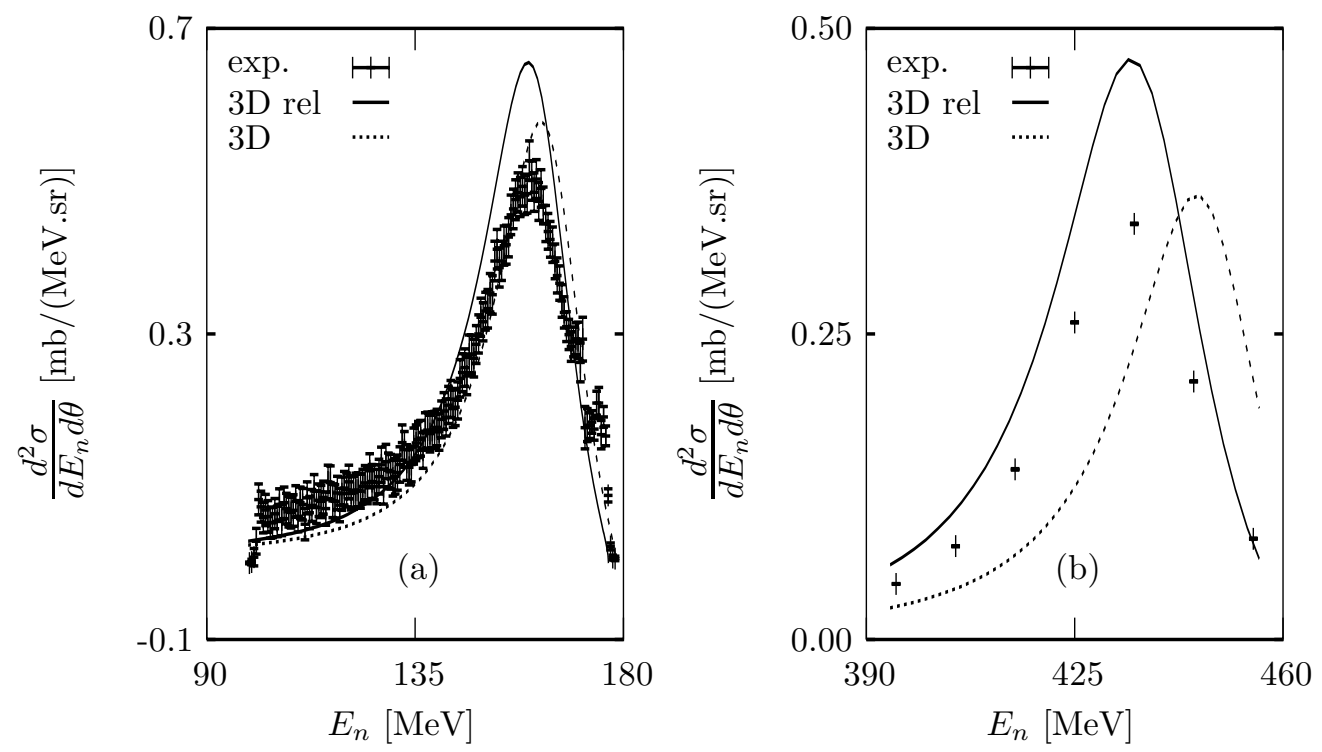

Figure 3. The spin averaged differential cross section at (a) $E_{l a b}=197 \mathrm{MeV}, \theta=24^{0}$ and (b) $E_{l a b}=495 \mathrm{MeV}, \theta=18^{0}$. The calculations are based on the Bonn-B potential, the data from Ref. [8] (a) and Ref. [6] (b).

\section{Summary and Conclusions}

We calculated the semi-exclusive pd break-up process within the Faddeev scheme in the leading order in a multiple scattering expansion in a $3 \mathrm{D}$ formulation based directly on momentum vectors. This technique has proved to be a viable alternative to traditional PW decompositions. For energies smaller than $200 \mathrm{MeV}$ our 3D calculations show perfect agreement with PW calculations. At energies larger than $200 \mathrm{MeV}$ the inclusion of relativistic kinematics proves essential to obtain the correct position of the quasi-free peak in the $(\mathrm{p}, \mathrm{n})$ charge exchange reaction.

\section{References}

1. L. D. Faddeev, Sov. Phys. JETP 12, 1014 (1961).

2. H. Liu, Ch. Elster, W. Glöckle, nucl-th/0410051 and to appear in Phys. Rev. C; Ch. Elster, et. al., Few-Body Systems 27, 83 (1999).

3. I. Fachruddin, Ch. Elster, W. Glöckle, Phys. Rev. C68, 054003 (2003).

4. I. Fachruddin, Ch. Elster, W. Glöckle, Phys. Rev. C62, 044002 (2000).

5. I. Fachruddin, Ch. Elster, W. Glöckle, Phys. Rev. C 63, 054003 (2001).

6. X. Y. Chen, et. al., Phys. Rev. C47, 2159 (1993).

7. T. Wakasa, et. al., Phys. Rev. C59, 3177 (1999).

8. D. L. Prout, et. al., Phys. Rev. C65, 034611 (2002).

9. R. B. Wiringa, V. G. J. Stoks, R. Schiavilla, Phys. Rev. C51, 38 (1995).

10. R. Machleidt, Adv. Nucl. Phys. 19, 180 (1989).

11. M. E. Rose, Elementary Theory of Angular Momentum (Wiley, New York, 1957).

12. R. Fong and J. Sucher, J. Math. Phys. 5, 456 (1964). 